Archives

$45 \mid 2010$

La preuve en histoire

\title{
Histoire, histoire ancienne
}

Réflexions sur le régime de la preuve. Tentative de refondation épistémologique ou reconfiguration disciplinaire?

\section{Charlotte Baratin}

\section{(2) OpenEdition}

Journals

Édition électronique

URL : http://journals.openedition.org/ccrh/3551

DOI : $10.4000 /$ ccrh.3551

ISSN : 1760-7906

Éditeur

Centre de recherches historiques - EHESS

Édition imprimée

Date de publication : 25 avril 2010

Pagination : $35-46$

ISSN : 0990-9141

\section{Référence électronique}

Charlotte Baratin, «Histoire, histoire ancienne », Les Cahiers du Centre de Recherches Historiques [En ligne], 45 | 2010, mis en ligne le 22 février 2012, consulté le 20 avril 2019. URL : http:// journals.openedition.org/ccrh/3551; DOI : 10.4000/ccrh.3551

Ce document a été généré automatiquement le 20 avril 2019.

Article L.111-1 du Code de la propriété intellectuelle. 


\section{Histoire, histoire ancienne}

Réflexions sur le régime de la preuve. Tentative de refondation épistémologique ou reconfiguration disciplinaire?

\section{Charlotte Baratin}

La vérité est personnelle.

Prends garde : tous ne sont pas dignes de la

confidence.

Accolade à celui qui, émergeant de sa fatigue et de

sa sueur,

S'avancera et me dira: " Je suis venu pour te

tromper ».

René Char ${ }^{1}$

\section{Des sciences sociales à l'histoire}

\section{Les débats sur la preuve : état des lieux}

1 Les débats suscités par la question de la "preuve», de l'«explication», de la « compréhension » dans les disciplines historiques et sociales sont particulièrement vifs dans les recherches épistémologiques contemporaines. Ils renvoient encore et toujours à la question de la nature de la scientificité de ces disciplines, sommées depuis le XIX ${ }^{\mathrm{e}}$ siècle de se positionner par rapport à une hiérarchie légitimiste d'origine philosophique qui fait des sciences expérimentales et formelles le paradigme scientifique par excellence. Après la remise en question des modèles statistiques et du primat de l'histoire de longue durée qui permettaient de rapprocher le type de connaissance produite de celles des sciences de la nature, le principe fécond d'un " retour au récit », annoncé une première fois par Paul Veyne dès 1970 avec son ouvrage Comment on écrit l'histoire ${ }^{2}$, et imposé dans le débat épistémologique par l'article désormais fameux de Laurence Stone ${ }^{3}$, est à son tour remis violemment en question par les tenants du «linguistic turn $»^{4}$. Ces derniers ont ranimé le débat sur la correspondance entre les représentations et les réalités objectives qu'elles désignent ${ }^{5}$. Dans les discussions en cours s'exprime l'exigence d'une véritable refondation épistémologique des sciences historiques et sociales. 
2 Parmi les différentes pistes proposées, Le raisonnement sociologique, de Jean-Claude Passeron, fournit une contribution essentielle ${ }^{6}$. Il y défend l'idée que les sciences historiques et sociales sont toujours confrontées à des configurations historiques particulières : elles ne raisonnent jamais à contexte constant ; ce caractère leur est propre et détermine la forme de leurs démonstrations. Les corrélations entre les éléments constitutifs de ces configurations varient à la fois dans le temps et dans l'espace ; il n'est pas possible d'en sélectionner des traits et de les reconstruire par l'expérimentation, afin d'observer des régularités qui permettraient de formuler des «lois» scientifiques. Paramètres et variables ne peuvent être isolés et contrôlés; ils se prêtent tout aussi mal à la quantification, à moins que l'on ne les extraie de leur contexte, alors que celui-ci seul leur donne sens. Force est donc, pour en rendre compte, d'élaborer des langages locaux, sectoriels et contextualisés. À la différence des sciences expérimentales et formelles, ni la démonstration formelle, ni le raisonnement expérimental à visée nomologique, ni le recours à une herméneutique universelle ne constituent des ressources adéquates pour l'élaboration des raisonnements des sciences historiques et sociales ni pour la construction de leurs résultats. Les raisonnements mis en œuvre ont recours à des méthodes d'administration de la preuve hétérogènes, que l'on associe dans un récit en langage naturel au sein duquel on les fait converger sémantiquement.

3 Au-delà du risque posé par l'atomisme méthodologique qu'elle permet de justifier, cette thèse fonde la possibilité - à première vue prometteuse - d'élaborer des épistémologies régionales; elle a en outre ceci de séduisant qu'elle donne un statut au recours à des éléments formels ou à une formalisation partielle des arguments dans la conduite des démonstrations. Si l'on en juge par les débats organisés dans les années quatre-vingt-dix par la Maison des Sciences de l'Homme à Paris autour de la notion de modèle, cette dernière donnée est particulièrement pertinente pour une discipline comme l'économie ou pour certaines branches de la sociologie qui ont recours à des outils formels d'origine mathématique ou statistique. L'architecture de l'ouvrage Le modèle et le récit, dirigé par Jean-Yves Grenier, Claude Grignon et Pierre-Michel Menger, qui rend compte d'une partie de ces débats ${ }^{7}$, suggère toutefois qu'ils ont abouti à une certaine aporie. La notion de modèle achoppe en effet sur son impropriété à rendre compte du vécu, et les usages empiriques qui en sont proposés ne permettent pas de répondre, sinon à la question de la preuve, du moins à celle de la légitimation des inférences, qui est précisément en jeu. Quant à l'histoire proprement dite, elle paraît bien faire la figure ainsi décrite par Jacques Rancière, bien qu'en l'occurrence il se soucie peu d'épistémologie : «Dans le concert des parents pauvres de la science, elle semblerait d'abord jouer le rôle de la plus pauvre cousine, vouée, malgré tout son appareil statistique, à toutes les approximations de la langue naturelle, les confusions de l'opinion et les séductions de la littérature ${ }^{8}$.

\section{La vérité comme construction sociale}

4 Giovanni Busino, dans un article de la Revue européenne des Sciences sociales ${ }^{9}$, prenant comme point de départ les études de Jean-Claude Passeron, a proposé d'observer les pratiques empiriques des chercheurs en sciences historiques et sociales pour " prouver » leurs assertions et valider leurs résultats. Réfléchir sur la notion de preuve permet en effet de mettre en évidence ce que les communautés de chercheurs considèrent comme une connaissance valide dans leur propre discipline. L'inventaire proposé par Giovanni Busino comprend la médecine, le droit, la préhistoire et l'archéologie, la géographie et la 
démographie, l'économie, la sociologie et, pour finir, l'histoire. De son analyse succincte des pratiques concrètes de chacune des disciplines, il conclut que l'on ne peut restituer aucune méthodologie cohérente et rigoureuse d'élaboration des assertions et de validation des connaissances. La logique de la preuve, telle qu'elle est formalisée en épistémologie des sciences, en lien avec le "dire vrai », semble donc inadéquate pour évaluer les raisonnements et les résultats des recherches en sciences sociales: en témoigne de façon particulièrement vive, outre les conflits internes aux disciplines, la pluralité d'interprétations possibles d'un même phénomène, dont on ne peut dépasser la juxtaposition concurrentielle. Giovanni Busino suggère que les sciences sociales soient désormais évaluées pour leur fonction propre : elles produisent des discours porteurs de sens et de significations à finalité pratique. Or si les significations peuvent être multiples, une seule et une seule à la fois peut être mobilisée en contexte ; le critère privilégié, selon lui, sera donc l'efficacité sociale de ces discours. Encore faudrait-il disposer de moyens d'identifier et de mesurer ce phénomène.

5 C'est en tout cas déplacer la question de la vérité historique : elle n'est plus alors fondée sur la correspondance entre le discours historique et les réalités objectives, mais considérée dans ses dimensions pratiques, comme un processus social. Marc Bloch dans son Apologie pour l'histoire a souligné le danger qu'il y avait pour son autonomie à légitimer l'histoire par son utilité sociale : c'est conforter l'idée qu'elle doit être au service d'un intérêt ou d'un pouvoir quelconque ${ }^{10}$. Il a pourtant été l'un des premiers à défendre l'idée que la vérité historique est une construction sociale : pour lui, en effet, un fait historique est vrai quand, soumis à l'épreuve de la réfutation collective, il est validé par les spécialistes du domaine considéré; c'est affirmer l'autonomie et l'autorité des communautés savantes dans la sphère publique.

6 Fort de ces thèses du grand médiéviste, Gérard Noiriel propose lui aussi de s'en remettre au jugement des pairs pour valider les thèses produites. C'est par la pédagogie que l'on tentera ensuite d'élargir la communauté de compétence au " grand public », de dépasser ce «malentendu entre la science telle qu'elle se fait ou aspire à se faire et le public qui lit », que déplorait déjà Marc Bloch ${ }^{11}$. Mais la position d'expert dans lequel se place ainsi l'historien, fondant la légitimité de son discours, en dernier ressort, sur un argument d'autorité garanti par une étroite communauté savante, est peu satisfaisante aujourd'hui, puisque cet argument d'autorité, justement, ne s'impose plus et que la discipline est sommée de justifier les fondements de ses assertions et de son statut scientifique. En outre, et ce n'est pas la moindre des objections, les remises en question, en l'occurrence, proviennent non pas tant, voire pas du tout, du public lettré mais bien des milieux savants eux-mêmes.

\section{Les historiens entre eux}

\section{Pour preuve : le corpus documentaire}

7 À la question de la vérité historique par la preuve, les historiens, en réalité, ont une réponse toute prête depuis la constitution de leur pratique en discipline à la fin du XIX siècle : le corpus documentaire et la méthode critique. Marc Bloch en faisait une question centrale de l'Apologie pour l'histoire et un argument décisif pour répondre à la question récurrente de la légitimité de l'histoire. L'établissement critique des sources et la réflexion sur la valeur du témoignage sont les instruments que les historiens se sont 
donnés pour élaborer leur propre règle de vérité ; les documents seuls permettent de trancher incontestablement la question du vrai et du faux en histoire.

8 Carlo Ginzburg, dans son ouvrage Le juge et l'historien, réflexions sur le rapport entre l'activité du juge et celle de l'historien développées en marge du procès d'Adriano Sofri, s'est imposé dans les récents débats : il s'exprime de façon très ferme contre la tendance fort répandue dans la recherche historique contemporaine - à la faveur, sans doute, de la fécondité de l'histoire des représentations - à refuser d'étudier le rapport de ces représentations avec les réalités objectives qu'elles désignent ou représentent ${ }^{12}$.

Nous savons bien que tout témoignage est construit selon un code déterminé : atteindre la réalité historique (ou la réalité) en prise directe est, par définition, impossible. Mais inférer de cela l'impossibilité de connaître la réalité signifie tomber par paresse dans une forme de scepticisme radical qui est à la fois insoutenable d'un point de vue existentiel et contradictoire du point de vue logique : comme on le sait, le choix fondamental du sceptique n'est pas soumis au doute méthodique qu'il prétend professer ${ }^{13}$.

Carlo Ginzburg affirme pour sa part avec vigueur que le métier d'historien repose sur « la possibilité de prouver, en fonction de règles déterminées, que $x$ a fait $y{ }^{14}{ }^{14}$. Et il rappelle l'origine de la constitution de l'histoire en discipline, lorsque collectionneurs et « antiquaires » apportèrent aux historiens d'alors le précieux concours des procédures de vérification et d'authentification qu'ils avaient éprouvées depuis longtemps pour répondre aux sceptiques. L'historien, donc, met en œuvre une méthode spécifique, celle que l'on a appelée autrefois la méthode historique; comme le rappelait HenriIrénée Marrou avec ferveur, il est animé par un souci de vérité, notion à laquelle celle de preuve est nouée par un lien historique. Il établit les faits que l'on peut connaître à partir des documents disponibles et, au-delà de ces faits explicitement avérés, il détermine ce que le contexte documentaire offre comme espace à la libre restitution et il oppose «le veto des sources " à une distorsion de la réalité objective. Le métier d'historien implique une "parenté » avec la vérité, pour reprendre librement les propos de Pierre VidalNaquet ${ }^{15}$.

10 Mais il y a un au-delà à ce socle de faits confirmés explicitement par les sources, qui entretient un rapport plus problématique avec la vérité. Carlo Ginzburg précise bien que contrairement au juge, l'historien, confronté à une connaissance lacunaire, se donne pour tâche d'exploiter des éléments connus du contexte pour proposer une restitution plausible des événements :

le contexte, entendu comme lieu de possibilités historiquement déterminées, sert à combler ce que les documents ne nous disent pas sur la vie d'une personne ${ }^{16}$.

Cette combinaison d'érudition et d'imagination est,

une tentative consciente de suggérer l'existence de dimensions historiques cachées, entre autres (mais pas seulement) parce que difficilement accessibles sur le plan de la documentation ${ }^{17}$.

12 L'historien aura souci de marquer la différence des plans dans le dispositif narratif, à la manière,

des restaurations modernes, où les lacunes d'un œuvre picturale ne sont plus cachées par la peinture mais soulignées par les techniques de comblement.

13 Car il ne faut pas perdre de vue que,

il s'agit là d'éventualités, pas de conséquences nécessaires; de conjectures, pas de faits avérés. En arriver à d'autres conclusions, c'est nier la dimension aléatoire et 
imprévisible qui constitue une part importante (fût-elle non exclusive) de la vie de

l'individu ${ }^{18}$. corps d'assertions distincts qui relèvent de deux domaines de raisonnement différents ${ }^{19}$. Il suggère, pour le démontrer, de schématiser dans une perspective logiciste les différentes opérations mobilisées dans nos raisonnements déductifs. Une grande partie d'entre elles, situées en amont du raisonnement, se traduisent alors sous la forme d'opérations de réécriture de type $p \Rightarrow q$, dont on réussit à circonscrire le domaine de recevabilité en leur associant suffisamment de facteurs explicites (qu'il appelle facteurs $\mathrm{C}$ : circonstances, critères, compléments, contexte géo-historique, croyances propres à une communauté particulière, common sense, etc.). Ces schématisations peuvent être éprouvées et reproduites, voire transposées dans une logique computationnelle pour être informatisées, ce qui permet, entre autres avantages, un travail plus rapide et l'intégration d'un plus grand nombre de données. Les propositions formelles ainsi produites devraient à terme pouvoir se passer tout à fait de leur glose en langage naturel : elles constituent un savoir proprement scientifique.

Jean-Claude Gardin tient ces schématisations pour des modèles inter-prétatifs; elles relèvent donc de la logique du modèle, par opposition à une logique du récit. En effet, « le critère le plus pertinent pour séparer le modèle et le récit réside dans « la décidabilité des formules $p \Rightarrow q$ mobilisées dans chacun d'eux, de part et d'autre de quelques seuils flottants ${ }^{20}$. Au-delà des propositions terminales de la schématisation s'ouvre le domaine des reconstructions relevant de l'indécidable, c'est-à-dire entre lesquelles on ne peut trancher sur des bases strictement empiriques: c'est le domaine du récit. La tâche de l'historien, le " jeu interprétatif », dit Jean Claude Gardin, se poursuit bien au-delà de ces seuils; elle relève alors de l'imagination historique, de la production d'hypothèses plausibles, de la recherche de significations. Un tel travail s'apparente à la littérature, mais il est strictement contrôlé :

nous avons toutes les raisons du monde de souhaiter que le jeu interprétatif se poursuive au-delà de ces seuils, au demeurant flottants, mais sur la base de propositions « scientifiques » établies en amont et par des moyens « littéraires » qui signalent le passage à des règles du jeu différentes, en aval ${ }^{21}$.

\section{De l'histoire vraie à l'histoire possible : droit de regard}

L'articulation étroite entre les propositions interprétatives de l'historien et un substrat de faits établis permet, à défaut de fonder en vérité toutes ses assertions, du moins d'avoir accès à leur fabrique. Cette forme de discours historique " mixte ", où un type de discours relaye l'autre, dans le texte ou dans les notes, s'est imposée tout à fait dans les usages savants. Un livre savant diffusé à un public plus large ne peut plus s'y soustraire non plus, même si les contraintes éditoriales en limitent les ressources. Mais si la forme nouvelle de combinaison d'énoncés - qu'elle innove ou constitue un retour provisoire à une histoire savante - modifie peu à peu le regard que l'on porte sur les productions des sciences humaines et incite à en adopter les exigences comme le souhaite Jean-Claude Gardin, on peut espérer qu'elle contribuera à imposer l'idée qu'un discours de vérité est toujours construit, et que tous peuvent avoir accès à ses fondements. Elle répond à l'exigence démocratique d'accès égal au savoir et préserve des dérives idéologiques comme des excès de pouvoir des figures d'expert ou d'autorité. N'est-ce pas contribuer, 
pour notre part, à faire de nous, comme le dit Antoine Prost, « les acteurs responsables de notre propre avenir $»{ }^{22}$, en nous redonnant les moyens d'innover?

\section{Positions théoriques : forme du récit et historicité}

17 Historiens et philosophes, public lettré peut-être, gardent pourtant la nostalgie d'une autre forme de discours, un discours qui n'aurait pas besoin de s'expliciter pour emporter la conviction. La production du soubassement «scientifique ", l'explicitation adjointe au discours, la glose ajoutée à la parole sont aussi bien le signe d'une crise de légitimité du discours, à laquelle elles fournissent une réponse toute provisoire.

Jean-Claude Perrot, dans les discussions qui eurent lieu à la Maison des Sciences de l'Homme sur la notion de modèle, situait le débat sur le plan de la rhétorique, considérant que la méfiance suscitée par les textes historiques pointait l'usage d'une rhétorique obsolète, qui ne fonctionnait plus ${ }^{23}$. Mais on peut douter qu'il s'agisse seulement de rhétorique. Ainsi Marc Bloch, dans l'une des ébauches qui subsistent de l'Apologie pour l'histoire, rappelait que :

le problème de la légitimité de l'histoire - car c'est bien de cela, au fond, qu'il s'agit - est, en soi, éternel. Mais il surgit régulièrement de façon particulièrement puissante, dans les époques troublées qui portent l'humanité à s'interroger sur ellemême et à scruter les moyens qu'elle peut posséder de percer les brumes du futur ${ }^{24}$

Le lien étroit, génétique, de la forme du discours historique avec une expérience propre du temps a été réaffirmé avec force par Jacques Rancière dans les Noms de l'histoire :

la question de la forme poétique selon laquelle l'histoire peut s'écrire est en effet strictement liée à celle du mode d'historicité selon lequel ses objets sont pensables 25.

20 Il fustige pour sa part l'expédient qui consiste à tenter de préserver le statut de vérité de son discours par "un surcroît de garanties scientifiques ou de rachat scientiste"; expédient dangereux et à contre emploi, puisqu'il ne fait qu'entretenir le soupçon qui pèse sur la validité du récit. Une fois encore, les historiens sont pris dans « la bataille séculaire avec le vieux mot d'histoire " : ils doivent élaborer le type de discours historique adapté à l'« historicité » de leur objet, susceptible de renouer dans sa forme avec le « triple contrat » de l'histoire : contrat scientifique, contrat narratif, et contrat politique enfin, qui lie les deux précédents aux contraintes qu'impose la configuration historique particulière dont ils expriment l'historicité.

\section{Retour à la pratique}

21 Il faut convenir que c'est là un programme un peu intimidant, que l'on peine à relier de façon immédiate à la tâche empirique quotidienne qui est la nôtre de rendre compte de nos corpus documentaires. Roger Chartier qui considère que ces considérations exigeantes, comme celles que formulent Paul Ricœur et Michel de Certeau dans leurs oeuvres, ont placé les historiens devant la nécessité d'une refondation épistémologique de leur discipline : Gérard Noiriel, lui, rappelle que la discipline s'est toujours développée à l'écart des débats épistémologiques. Il suggère qu'il serait plus fructueux, « au lieu de prolonger le débat sur la nature de l'histoire ", «de concentrer notre effort sur l'étude des pratiques et des activités de recherches ${ }^{26}$. Les historiens, et Roger Chartier lui- 
même, ne cessent en effet d'expérimenter des méthodes et des problématiques et c'est par une démarche pragmatiste qu'ils contribuent au renouvellement de leur discipline.

\section{De l'histoire à l'histoire ancienne et retour}

\section{La pénurie dans l'abondance : mise en débat}

Voyons donc, pour notre part, du côté de l'histoire ancienne, puisque c'est là, comme l'a rappelé Carlo Ginzburg, que s'est exprimée d'abord l'exigence de "prouver» les assertions des historiens, et là que Jean-Claude Gardin semble pouvoir expérimenter son programme logiciste. Ce domaine est-il à l'abri des débats de l'épistémologie contemporaine ? D'emblée on pourrait se croire dans un autre pays, l'heureux pays peutêtre de Jean-Claude Gardin. Les sources archéologiques, en effet, sont en accroissement exponentiel depuis deux générations. Le progrès considérable des techniques de prospection, de fouille, de traitement des données a mis à la disposition des historiens une quantité considérable d'informations nouvelles. Il y règne un climat positiviste tout à fait rafraîchissant, né du contact renouvelé avec les "réalités objectives ", et renforcé de l'assurance que les hypothèses formulées trouveront à s'enrichir, pourront être vérifiées ou invalidées par des données nouvelles: l' «observation dirigée » qu'évoquait JeanClaude Gardin peut y avoir valeur d'expérimentation.

Pour autant, comme l'a rappelé avec force Moses Finley, malgré l'euphorie de l'abondance nouvelle, la pénurie demeure de règle pour l'historien, car le corpus de sources écrites primaires reste à peu près constant : «La rareté des sources littéraires primaires est une malédiction pour l'histoire ancienne toute entière! ${ }^{27}$. Les documents de provenance archéologique constituent un échantillon dispersé dans le temps et dans l'espace, souvent dépourvu de contexte, dont on ne peut restituer la représentativité quelle que soit la taille. Dans la plupart des domaines, en l'absence de tout exposé narratif contemporain, les «mises en intrigue» sont proprement impossibles. Les historiens sont donc le plus souvent cantonnés à faire une histoire, souvent lacunaire, de leur support documentaire ou des phénomènes qui en dépendent directement. C'est ainsi que se sont considérablement renouvelées les histoires de l'art et des techniques, du fait monétaire ou du paysage, dont les sources documentaires elles-mêmes constituent l'objet d'étude, renouvelées aussi l'histoire fondée sur les sources littéraires, histoire des idées, des représentations, de la rhétorique, de la littérature, de l'historiographie.

L'illustration la plus claire de ces phénomènes est la reconfiguration des départements universitaires d'histoire ancienne sous le nouveau nom de "Sciences de l'Antiquité » ou bien, mieux, "Sciences des mondes anciens ", associant les divers spécialistes sous la dénomination de leur spécialité documentaire. On ne peut que déplorer que le nom d'«historien" ne soit plus revendiqué - ou octroyé - qu'aux spécialistes des sources littéraires. Mais ces querelles de chapelles recouvrent un débat particulièrement important sur la nature et l'usage des sources anciennes, " un débat plus large et plus délibéré qu'il n'y en eut jamais auparavant depuis, disons, le début du XIX siècle, le temps de Boeckh et de Niebuhr " affirme Moses I. Finley ${ }^{28}$. Et ce que reflète ce débat, outre l'augmentation des corpus documentaires, c'est l'apparition de nouveaux intérêts, la considération de nouveaux objets, la formulation de nouvelles questions historiques. 


\section{Nouveaux objets}

Le renouvellement le plus considérable des études anciennes est la remise en question du découpage spatio-temporel dévolu à l'histoire ancienne. La notion d'Antiquité coïncide en effet avec l'espace désigné plus ou moins partiellement par le corpus de textes grécoromains et avec les époques qu'ils évoquent. À ce découpage étaient associés tous les partis pris véhiculés par ces textes, dont les sources ne permettaient pas de prendre la mesure et dont la valeur de référence culturelle avait cristallisé les connotations. Considérer le territoire de la domination romaine comme celui du monde civilisé n'était pas le moindre de ces partis pris. Or les développements de l'archéologie ont permis de s'affranchir de ces bornes arbitraires, d'en explorer les confins, renouvelant du tout au tout l'optique des descriptions et catégories classificatoires d'origine gréco-romaine. C'est ainsi qu'ont été explorés l'Afrique conquise, l'Iran préislamique conquis par Alexandre le Grand, occupé ensuite par les Séleucides, les Parthes et les Sassanides, les régions d'Europe centrale et orientale, la région de la mer Noire et les zones steppiques jusqu'à la Chine, les territoires indiens. Si les synthèses sont malaisées, restent fortement hypothétiques et n'ont souvent de réelle valeur qu'heuristique, ces recherches, qui peinent à s'imposer dans les départements de recherche universitaire, exigent de définir de nouveaux ensembles pertinents et de réfléchir à la notion de contacts, de transferts, de réseaux, d'échanges dynamiques. En 1978 déjà, Yvon Thébert, à propos de l'Afrique romanisée, écrivait :

L'un des problèmes de base consiste donc à élaborer des "blocs historiques », fondés sur un espace et une durée cohérents, et au sein desquels s'articuleraient les différents types de recherche, articulation dont il est logique de penser qu'elle variera suivant les périodes et les régions considérées, en fonction à la fois des problèmes qui se posent et de la nature de la documentation historique. Un des avantages de la définition de ces "blocs", de ces secteurs de recherche, serait d'éviter deux types de fausses autonomies déjà signalées: le morcellement au niveau des spécialités, qui ne se fondent que sur la prétention de certains auteurs d'ennoblir leur discipline en lui donnant une indépendance qui s'accompagne du sentiment de sa supériorité, et le morcellement au niveau des sujets, qui aboutit à découper, sans aucune justification historique, ce dont le trait dominant est d'avoir fait partie d'un ensemble ${ }^{29}$.

Le puissant écho aux débats contemporains suscités dans les milieux de recherche anglosaxons par l'«histoire globale», l'« histoire connectée», l'histoire transnationale, l'anthropologie dite multi sites, qui renouvellent les catégories analytiques classiques, bouleversent les échelles d'analyse et modifient substantiellement les grands récits de l'invention des modernités, suggère que c'est sans doute dans cette direction que quelque chose s'invente.

\section{Conclusion}

Si l'on en croit Gérard Noiriel, c'est par leurs pratiques empiriques que les historiens, depuis toujours, ont répondu aux remises en question épistémologiques. Arnaldo Momigliano $^{30}$ a mis de telles remises en question à l'origine même de la constitution de l'histoire ancienne comme discipline : c'est la confrontation avec les sceptiques d'abord, puis les philosophes des Lumières, qui a suscité l'association fructueuse des «Antiquaires» et des historiens traditionnels qui a donné naissance à la «méthode 
historique actuelle ». L'élaboration d'une méthodologie historique rigoureuse, fondée sur la combinaison entre sources écrites et sources matérielles, fut la réponse aux sceptiques; à l'exigence philosophique d'élaborer une vision historique de plus grande ampleur répondit l'historicisation des différents aspects de civilisation qui n'entraient pas dans le cadre strict de l'histoire politique traditionnelle - l'art, le droit, les institutions, la religion, etc. - tandis qu'apparaissaient sur la scène historique des peuples comme les Étrusques qui n'avaient pas de place dans les textes.

Proposons une autre lecture: n'est-ce pas plutôt un premier refus de l'emprise d'un certain discours historique, histoire politique à l'usage des princes, fondée exclusivement sur les textes, que signalait le mouvement sceptique ? La réponse de type "scientiste» avant l'heure à cette première injonction sociale a davantage servi aux historiens d'outil heuristique, qu'offert aux sceptiques des gages de véracité. De fait, elle n'a nullement suffi à fonder un nouveau régime de vérité. Elle $\mathrm{a}$, en revanche, permis d'explorer de nouveaux domaines qui entraient en résonance avec les préoccupations du temps, la mise en place d'un état de droit et de règlements démocratiques; mais surtout, tandis que s'inventait en Europe un récit fondateur pour les nations nouvelles, le discours historique s'est enraciné dans le terroir antique dont les archéologues découvraient les richesses.

Dans les débats contemporains sur la preuve, suscités par le soupçon que le discours historique manque son but, à savoir la réalité historique vers laquelle il tend, ce qui est en jeu n'est-il pas de la même façon la remise en question d'un certain type de discours historique, de ses objets de prédilection, de ses partis pris, celui dont nous héritons des Annales et de la Nouvelle Histoire ? C'est, appliquée à l'historiographie de la révolution française, la thèse de Jacques Rancière dans les Noms de l'histoire ${ }^{31}$. C'est ce que suggère aussi le renouvellement considérable des objets historiques et des échelles d'analyse dans tous les domaines de la discipline, né à la faveur des défis lancés par les sceptiques et de l'exigence posée par les philosophes.

\section{NOTES}

1. René Char, « Rougeurs des matinaux », in Matinaux, Gallimard, 1950.

2. Paul Veyne, Comment on écrit l'histoire, Paris, Seuil, 1971.

3. Laurence Stone, "Revival of Narrative. Reflections on an Old New History ", Past and Present, vol. 85, 1979. Sur ce « retour du récit », les travaux qui s'en réclament, les critiques suscitées, voir Gérard Noiriel, "Le retour du récit et ses critiques. De Laurence Stone à Roger Chartier ", in Penser avec, penser contre. Itinéraire d'un historien, Paris, Belin, 2003, p. 99-117.

4. Sur ce mouvement qui a suivi le «retour au récit» et qui en fait une interprétation épistémologique radicale, voir Gérard Noiriel, op.cit., p. 109-113.

5. Paul Veyne, Comment on écrit l'histoire, Paris, Seuil, 1971; Laurence Stone, "Revival of Narrative. Reflections on an Old New History », Past and Present, vol. 85, 1979. Sur ce " retour du récit », les travaux qui s'en réclament, les critiques suscitées, voir Gérard Noiriel, « Le retour du récit et ses critiques. De Laurence Stone à Roger Chartier», in Penser avec, penser contre. Itinéraire d'un historien, Paris, Belin, 2003, p. 99-117. 
6. Jean-Claude Passeron, Le raisonnement sociologique. L'espace non-poppérien du raisonnement naturel , Paris, Nathan, 1991.

7. Le modèle et le récit, Jean-Yves Grenier, Claude Grignon, Pierre-Michel Menger (dirs.), Paris, Maison des Sciences de l'Homme, 2001.

8. Jacques Rancière, Les Noms de l'Histoire. Essai de Poétique du savoir, Paris, Seuil, 1992, cit. p. 23.

9. Giovanni Busino, «La preuve dans les sciences sociales», Revue européenne des sciences sociales, t. XLI-128, p. 11-61.

10. Marc Bloch, Apologie pour l'histoire ou Métier d'historien, Paris, Colin, 1993 [1949, posthume].

11. Gérard Noiriel, op.cit., p. 75-76.

12. Carlo Ginzburg, Le juge et l'historien. Considérations en marge du procès Sofri, Lagrasse,Verdier, 1997.

13. Carlo Ginzburg, op. cit., p. 22.

14. Ibid., p. 23.

15. Pierre Vidal-Naquet, Le choix de l'Histoire: pourquoi et comment je suis devenu historien, Paris, Arléa, 2007 [2004] à propos de ses premiers engagements politiques: «J'entendais ne jamais oublier quel était mon métier et qu'il impliquait une fraternité, une parenté (en grec syngeneia) avec la vérité », p. 33.

16. Carlo Ginzburg, op. cit, p. 116.

17. Ibid., p. 111.

18. Carlo Ginzburg, op. cit., p. 116 ; il y commente l'ouvrage de Nathalie Davies, Le retour de Martin Guerre, Paris, Taillandier, 2008.

19. Jean-Claude Gardin, «Entre modèle et récit : les flottements de la troisième voie ", Le modèle et le récit, op.cit., p. 457-487.

20. Jean-Claude Gardin, op. cit., p. 479.

21. Ibid., p. 481.

22. Antoine Prost, Douze leçons sur l'histoire, Paris, Seuil, 1996, p. 306.

23. Jean-Claude Gardin, "Lecture critique ", Cahiers du séminaire "Le modèle et le récit", INRAMaison des Sciences de l'Homme, Paris, 1997, p. 18.

24. Voir Marc Bloch, op. cit., «Reliquat probable des réflexions sur l'histoire écrites à Rennes en Juin $1940 »$, p. 60.

25. Jacques Rancière, op. cit., p. 204.

26. Gérard Noiriel, op. cit., p. 116-117.

27. Moses I. Finley, Sur l'histoire ancienne. La matière, la forme et la méthode, Paris, La Découverte, 1987.

28. Moses I. Finley, op. cit., p. 47.

29. Yvon Thébert, « Romanisation et déromanisation en Afrique : histoire décolonisée ou histoire inversée ?", Annales. Économies, Sociétés, Civilisations, 1978, vol. 33, n 1, p. 64-82, part. p. 73.

30. Arnaldo Momigliano, « Ancient History and the Antiquarian », in Id., Contributo alla storia degli studi classici, Rome, Edizioni di Storia e Letteratura, 1955, p. 67-106.

31. Jacques Rancière, op. cit. 


\section{AUTEUR}

CHARLOTTE BARATIN

ENS Ulm 